\title{
Secret professionnel et dangerosité
}

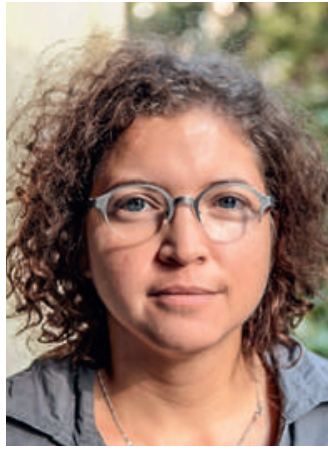

Samia Hurst
L'évaluation de la dangerosité des détenus émeut la Suisse romande, qui a connu deux tragédies avec les meurtres de Marie, tuée par un détenu aux arrêts domiciliaires avec lequel elle entretenait une relation, et d'Adeline, une sociothérapeute qui accompagnait en sortie autorisée un détenu qui l'a tuée pour prendre la fuite. Devant l'indignation suscitée par ces affaires, les élus cherchent des solutions pour limiter les risques futurs. Une tâche exigeante, dont tous reconnaissent l'importance.

Malheureusement, la solution proposée n'est pas à la hauteur. Sur recommandation de la Conférence latine des chefs des départements de justice et police, Genève, Vaud, et le Valais proposent de soumettre les professionnels de la santé à une obligation de transmettre les informations considérées comme pertinentes pour l'évaluation de la dangerosité d'un détenu. Cette évaluation est difficile. Même si le secret professionnel n'a jamais été mis en cause lors des audits qui ont suivi les deux affaires, on peut comprendre ces tentatives d'avoir plus d'information pour faire mieux.

\section{«La confidentialité est un outil de la médecine, aussi indispensable qu'un stéthoscope ou un bistouri.»}

Cette «solution» repose pourtant sur de profonds malentendus. Le secret professionnel sert à protéger le droit à la sphère privée du patient. Il est donc présenté ici comme en tension avec la protection des victimes potentielles. Mais ces victimes sont en fait elles aussi protégées par le respect du secret professionnel en prison. La confidentialité est un outil de la médecine, aussi indispensable qu'un stéthoscope ou un bistouri. La limiter limite la possibilité même de la thérapie. Imaginez que votre médecin divulgue ce que vous lui confieriez. Vous lui confieriez nettement moins. Vous pourrez certes encore vous «laisser soigner» [1], mais dans une psychothérapie cela reviendrait à rester passif et en limiterait fortement l'efficacité.

Dans les cas concernés, limiter la possibilité de la thérapie ne limite pas seulement l'accès aux soins des détenus. Ce serait déjà grave, car une sentence de prison n'est pas censée inclure un déni de médecine. Mais ici la thérapie sert aussi à la protection des victimes. Le code pénal prévoit des mesures thérapeutiques précisément parce que l'on compte sur elles pour diminuer la dangerosité. Ordonner des mesures thérapeutiques tout en limitant le secret professionnel, c'est vouloir le beurre et l'argent du beurre.

Il faut comprendre aussi que l'information concernée n'aurait pas de limites claires. Les médecins ont déjà la responsabilité de divulguer les infor-

\section{«Cette mesure sacrifierait leur rôle de thérapeutes.»}

mations nécessaires pour protéger des tiers, directement dans l'urgence ou en se faisant délier du secret dans les autres cas [2]. L'obligation considérée ici vise à avoir plus d'information, mais évaluer la dangerosité restera difficile, même avec toute l'information. En aurait-on déjà davantage ainsi? Il faudrait que les détenus se confient comme avant à leurs thérapeutes alors que tout ce qu'ils diraient pourrait être retenu contre eux.

Soumettre les professionnels de la santé à cette nouvelle obligation d'information serait donc contraire non seulement aux droits des détenus mais aussi à la protection des victimes. Cela n'améliorerait pas l'évaluation de la dangerosité. Cela contraindrait les médecins à traiter ces détenus, entre autres pour protéger la population, sans avoir les moyens d'être efficaces: un rôle d'alibi qu'on les imagine mal accepter sans autres. Cette mesure sacrifierait leur rôle de thérapeutes pour en faire des experts, sans leur en donner ni la formation ni les moyens et alors que des experts formés existent déjà. Ceux qui élevent la voix pour s'y opposer ont donc raison [3, 4]; espérons que d'autres comprennent à quel point.

Samia Hurst *

\section{Références}

1 Poggia M. La vie des victimes vaut davantage que le secret des confidences. Le Temps. 201416 avril.

2 Dumoulin JF. Le secret professionnel des soignants et leur obligation de témoigner selon les nouveaux codes de procédure fédéraux. Jusletter. 18 janvier; 2010.

3 Matter M. Le secret médical est intangible. Bull Méd Suisses. 2014; 95(18):681.

4 Sprumont D. Secret médical en prison: notre sécurité mise en cause. Le Temps. 8 avril; 2014. 